\title{
How the Post-Fracture Rehabilitation Choice Affects Brain Function in Older People?
}

\author{
Noriko Mitsuboshia, ${ }^{a}$ Minoru Kouzukia Shigeru Mochida ${ }^{b}$ \\ Kanehito Morimoto $^{\text {b }}$ Katsuya Urakami ${ }^{a}$ \\ a Department of Biological Regulation, School of Health Science, Faculty of Medicine, \\ Tottori University, Yonago, Japan; ' $Y u k o u k a i$ Kaikeonsen Hospital, Yonago, Japan
}

Keywords

Falls · Electroencephalogram · Cognition · Oxidative stress · Plasticity

\begin{abstract}
Background: We investigated how the type of rehabilitation affects brain function and antioxidant potential. Methods: Twenty-eight patients hospitalized for fall-related fractures were assigned to either a physical therapy group or an occupational therapy group. Cognition was assessed using the Touch Panel-type Dementia Assessment Scale (TDAS) and oxidative stress with serum pentosidine levels. Spectral analysis and coherence analysis were also performed. Results: Changes in TDAS scores and serum pentosidine levels did not differ significantly between the 2 therapies. Power spectral analysis revealed a significant intergroup difference in $\delta$ waves. Coherence analysis showed significant intergroup differences in the activities of $\delta$ waves and $\beta$ waves. Conclusions: Cognitive function and antioxidant potential did not differ between the 2 types of rehabilitation. However, the impact on cerebral neuronal activity may have differed.

(C) 2019 The Author(s)

Published by S. Karger AG, Basel

\section{Introduction}

Deterioration not only of motor function but also of dual-task performance has been associated with falls in older people [1]. Body movements are seldom completed with a single motion. We need to allocate and balance our attention to dual tasks to carry out each task appropriately $[2,3]$. The frontal association cortex is believed to play a major role in this function although available attention is limited [2]. Thus, we must perform tasks by allocating our limited resources to the right target.
\end{abstract}


Brittle bones predispose older patients to bone fractures in a fall. Spinal vertebral fracture and proximal femur fracture are the most likely to occur [4, 5]. These fractures cause deterioration of both activities of daily living and quality of life [6-8]. Patients hospitalized with fractures undergo rehabilitation to help them resume their ADL, to prevent becoming bedridden, and to allow them to return to their homes and social circles as early as possible. Rehabilitation is available in 2 forms, i.e., physical therapy (PT) and occupational therapy (OT). In many cases, patients with fall-related fractures undergo PT to recover/maintain fundamental movement skills and to prevent worsening of them.

Exercises involving only a single movement such as standing up or walking will not lead to improved daily life skills. Therefore, OT should be combined with PT to help maintain and improve the patient's ability to apply movements and adapt to society by facilitating tasks such as eating, toileting, and bathing.

Exercise is believed to activate acetylcholine release and to promote the proliferation of neural stem cells [9]. Acetylcholine alters information transmission in the cerebral cortex, enhancing activity [10]. Electroencephalograms (EEG) allow changes in electrical potentials during the transmission of information to be visualized. Changes in frequency, amplitude, and spatial coherence in the brain are mainly brought about by changes in neurotransmitters [11]. The complicated neural network observed in the mammalian cerebral cortex has evolved as a well-balanced network between the excitatory glutamatergic projection neuron (pyramidal cell) and the inhibitory $\gamma$-aminobutyric acid (GABA) neuron (interneuron) [12]. Pyramidal cells compose the basic structure of nerve cells which are specialized in transmitting information between different cerebral cortex areas [13]. Interneurons transmit inhibitory signals to pyramidal cells to prevent them from going out of control. By numerically processing the electrical potential of brain waves, we can assess neural activity with power spectral analysis and neural synchronization with coherence analysis [14]. Synchronization of EEG is considered an index of the functional connectivity of the intrabrain network, and it is associated with age-related deterioration of cognitive function. Connectivity between the frontal lobe and the occipital lobe of the brain is particularly important and believed to predict the risk of developing dementia [15].

Aging is the greatest risk factor for deterioration of cognitive function and promotes the accumulation of oxidative stress in the brain [16]. To evaluate oxidative stress, advanced glycation end-products resulting from the nonenzymatic reactions between glucose and protein in living cells are used as markers [17]. Moderate daily exercise has been reported to increase antioxidative enzymes $[18,19]$ and to enhance the ability to defend against oxidative stress [20]. Increased bone density and improved bone quality are believed to protect older people from osteoporosis which predisposes them to fractures [21]. Thus, we measured serum pentosidine levels, which are considered bone quality markers among the oxidative stress markers. If we compare the structure of bone to that of reinforced concrete buildings, the bone mass corresponds to the concrete sections, the bone quality to the reinforced steel, and pentosidine to "rust" on the reinforced steel [22].

In this study, we investigated whether brain function and antioxidant power would differ in patients hospitalized for rehabilitation following fall-related fractures in a PT group and a PT+OT group.

\section{Materials and Methods}

\section{Participants}

Of the 34 patients who were admitted to the convalescent rehabilitation ward of the Medical Corporation Yukoukai Kaikeonsen Hospital between June 1, 2017, and March 31, 2018 , for hip or vertebral fractures who had been diagnosed at admission by an attending 
doctor as needing treatment for more than 1 month, 6 patients who met the exclusion criteria were ineligible and the remaining 28 patients were enrolled as study participants. Excluded were those whose fractures were caused by a strong external force, or who had severe complications other than bone fractures, or who were judged by a doctor to be unsuited to participate in this study.

\section{Convalescent Rehabilitation}

Hospitalization in the convalescent rehabilitation ward was limited to a maximum of 3 months. The study participants were randomly assigned to 1 of 2 treatments at hospitalization, i.e., a PT group and a PT+OT group. Patients in the PT group received 120 min of PT every day during hospitalization, while those in the PT+OT group received $60 \mathrm{~min}$ of PT and 60 min of OT. Rehabilitation continued until they were discharged from the hospital, and more than $80 \%$ of the participants completed the rehabilitation therapy.

\section{Primary Endpoints}

To evaluate the effectiveness of rehabilitation, the following items were determined both before (within 1 week after hospitalization) and after intervention (no later than 1 week before the planned hospital discharge date).

\section{Cognitive Assessment}

The Touch Panel-type Dementia Assessment Scale (TDAS) was used for cognitive assessment [23]. It was developed by modifying the Alzheimer's Disease Assessment Scalecognitive subscale (ADAS-cog) [24], a scale to assess AD patients' cognitive function, and by equipping it with a touch panel system that enabled us to conduct a test in a shorter period of time (shortened from 60 to $20 \mathrm{~min}$ ) even without expert assistance. The following 9 test items were included: following a command, visual-spatial perception, accuracy of the order of a process, naming fingers, orientation, money calculation, object recognition, and reading a clock face (of a nondigital clock). A total score of 0 was defined as perfect.

More incorrect answers meant higher scores. When all answers were incorrect, the score was 101 points.

Blood Tests

Measurement of serum pentosidine levels was outsourced to Falco Holdings Co., Ltd. (Kyoto, Japan).

\section{Electroencephalography}

Using the Neurofax EEG-1214 system (NIHON KOHDEN CORPORATION, Tokyo, Japan), EEG were recorded. The EEG was recorded in a 5-min period in subjects in the wake resting state from 19 electrodes positioned according to the International 10-20 system (i.e., Fp1, Fp2, F3, Fz, F4, F7, F8, T3, T4, C3, Cz, C4, T5, T6, P3, Pz, P4, 01, and 02). Data were sampled at $500 \mathrm{~Hz}$ and band pass filtered at $30 \mathrm{~Hz}$. The status of handedness for every participant was right-handedness. Special attention was devoted to obtaining maximal relaxation and cooperation of the subjects, obtaining high-quality EEG recordings, and avoiding well-known effects of stress and anxiety.

One epoch was defined as $2 \mathrm{~s}$ of EEG free of gross body movement-induced artifacts [25]. MATLAB R2017b was used to analyze data. We analyzed 10 epochs of EEG. Fp1 and Fp2 were excluded because of eye movements. Fast Fourier transformation was performed [26] to calculate the absolute value of the power spectrum at each frequency at each electrode, with each frequency defined as follows: $\delta$ wave, $0.5-3.5 \mathrm{~Hz} ; \theta$ wave, $3.5-7.5 \mathrm{~Hz} ; \alpha-1$ wave, 7.5-9.5; $\alpha-2$ wave, 9.5-12.5 Hz; $\beta-1$ wave, $12.5-17.5 \mathrm{~Hz}$; and $\beta-2$ wave, $17.5-25.0 \mathrm{~Hz}$. The power of the 
Table 1. Patient characteristics

\begin{tabular}{lccc}
\hline & $\begin{array}{l}\text { PT group } \\
(n=16)\end{array}$ & $\begin{array}{l}\text { PT+OT group } \\
(n=12)\end{array}$ & $p$ value \\
\hline Male/female ratio & $2 / 14$ & $4 / 8$ & 0.354 \\
Vertebral fracture/hip fracture ratio & $11 / 5$ & $4 / 8$ & 0.121 \\
Age, years & $85.1 \pm 6.4$ & $84.7 \pm 6.9$ & 0.641 \\
Length of stay, days & $71.1 \pm 18.6$ & $76.4 \pm 13.6$ & 0.568 \\
\hline
\end{tabular}

Data are presented as means \pm SD.

Table 2. TDAS and serum pentosidine levels before and after intervention

\begin{tabular}{llllllll}
\hline & \multicolumn{2}{l}{ PT group $(n=16)$} & \multirow{2}{*}{$p$ value } & & \multicolumn{2}{l}{ PT+OT group $(n=12)$} & $p$ value \\
\cline { 2 - 3 } & before & after & & & before & after & \\
\hline TDAS & $18.25 \pm 14.54$ & $10.88 \pm 10.05$ & 0.003 & & $16.83 \pm 13.48$ & $12.58 \pm 13.40$ & 0.026 \\
Pentosidine & $0.067 \pm 0.019$ & $0.051 \pm 0.019$ & 0.011 & & $0.074 \pm 0.032$ & $0.053 \pm 0.017$ & 0.025 \\
\hline
\end{tabular}

Data presented as means \pm SD.

$\delta$ waves, $\theta$ waves, $\alpha-1$ waves, $\alpha-2$ waves, $\beta-1$ waves, and $\beta-2$ waves in each electrode were averaged to calculate the absolute power. The absolute power at each electrode site was used to calculate the relative power, which indicated how much each electrode contributed to the total [26].

To calculate synchronization between the frontal and occipital lobes, synchronization between F3-F4, 01-02, and P3-P4 was defined as interhemispheric and synchronization between F3-P3, F4-P4, F4-T6, F3-T5, F3-01, and F4-02 was defined as intrahemispheric. Coherence values in each frequency zone were calculated [15].

\section{Statistical Analysis}

Kolmogorov-Smirnov was used to test for normality. An unpaired $t$ test and Fisher's exact test were used to compare basic patient characteristics between the 2 groups. The Wilcoxon signed-rank test was used to compare data before and after intervention. Analysis of covariance was used to compare changes between the 2 groups before and after intervention with adjustments for gender, fracture site, and age. $p<0.05$ was considered statistically significant. IBM SPSS Statistics version 25.0 was used for statistical analysis in this study.

\section{Results}

\section{Participants}

Patient characteristics at baseline are shown in Table 1. No significant difference in age, breakdown of fracture sites, or male-to-female ratios were found between the 2 groups.

\section{Changes before and after Intervention}

Touch Panel-Type Dementia Assessment Scale

Table 2 shows the changes in TDAS before and after rehabilitation. TDAS scores improved significantly after both types of interventions. As shown in Table 3, however, TDAS changes 
Table 3. Changes in TDAS, pentosidine, and EEG power and coherence before and after intervention (i.e., PT and PT+OT): results of analysis of covariance

\begin{tabular}{|c|c|c|c|c|c|c|}
\hline & \multirow{2}{*}{$\begin{array}{l}\text { PT } \\
(n=16)\end{array}$} & \multirow{2}{*}{$\begin{array}{l}\mathrm{PT}+0 \mathrm{~T} \\
(n=12)\end{array}$} & \multirow[t]{2}{*}{$p$ value } & \multicolumn{3}{|c|}{$p$ value for covariates } \\
\hline & & & & gender & fracture site & age \\
\hline TDAS & $-6.956 \pm 8.371$ & $-4.808 \pm 5.952$ & 0.576 & 0.387 & 0.460 & 0.361 \\
\hline Pentosidine & $-0.024 \pm 0.025$ & $-0.014 \pm 0.020$ & 0.399 & 0.733 & 0.310 & 0.957 \\
\hline \multicolumn{7}{|c|}{ Power spectrum } \\
\hline$\delta$ & $-0.138 \pm 0.141$ & $0.000 \pm 0.075$ & 0.030 & 0.326 & 0.020 & 0.260 \\
\hline$\theta$ & $0.005 \pm 0.090$ & $-0.017 \pm 0.050$ & 0.580 & 0.835 & 0.342 & 0.388 \\
\hline$\alpha-1$ & $0.100 \pm 0.130$ & $0.004 \pm 0.055$ & 0.087 & 0.563 & 0.039 & 0.052 \\
\hline$\alpha-2$ & $0.004 \pm 0.104$ & $0.004 \pm 0.050$ & 1.000 & 0.448 & 0.496 & 0.186 \\
\hline$\beta-1$ & $0.019 \pm 0.018$ & $-0.002 \pm 0.022$ & 0.054 & 0.587 & 0.026 & 0.898 \\
\hline$\beta-2$ & $0.011 \pm 0.030$ & $0.010 \pm 0.024$ & 0.935 & 0.516 & 0.260 & 0.268 \\
\hline \multicolumn{7}{|l|}{ F8 } \\
\hline$\delta$ & $-0.132 \pm 0.166$ & $0.029 \pm 0.102$ & 0.037 & 0.479 & 0.182 & 0.080 \\
\hline$\theta$ & $0.014 \pm 0.091$ & $-0.007 \pm 0.044$ & 0.581 & 0.430 & 0.864 & 0.244 \\
\hline$\alpha-1$ & $0.080 \pm 0.117$ & $-0.009 \pm 0.055$ & 0.082 & 0.634 & 0.068 & 0.143 \\
\hline$\alpha-2$ & $0.013 \pm 0.091$ & $-0.012 \pm 0.051$ & 0.533 & 0.200 & 0.577 & 0.335 \\
\hline$\beta-1$ & $0.012 \pm 0.027$ & $-0.003 \pm 0.028$ & 0.270 & 0.959 & 0.233 & 0.425 \\
\hline$\beta-2$ & $0.012 \pm 0.027$ & $0.002 \pm 0.034$ & 0.500 & 0.654 & 0.644 & 0.618 \\
\hline \multicolumn{7}{|l|}{$\mathrm{T} 4$} \\
\hline$\delta$ & $-0.137 \pm 0.141$ & $0.058 \pm 0.082$ & 0.004 & 0.140 & 0.152 & 0.198 \\
\hline$\theta$ & $0.024 \pm 0.102$ & $-0.018 \pm 0.075$ & 0.374 & 0.522 & 0.612 & 0.946 \\
\hline$\alpha-1$ & $0.077 \pm 0.080$ & $-0.025 \pm 0.060$ & 0.011 & 0.058 & 0.037 & 0.013 \\
\hline$\alpha-2$ & $0.026 \pm 0.052$ & $-0.015 \pm 0.034$ & 0.089 & 0.041 & 0.157 & 0.687 \\
\hline$\beta-1$ & $0.011 \pm 0.031$ & $0.003 \pm 0.031$ & 0.592 & 0.941 & 0.832 & 0.589 \\
\hline$\beta-2$ & $-0.001 \pm 0.046$ & $-0.003 \pm 0.053$ & 0.942 & 0.974 & 0.842 & 0.504 \\
\hline \multicolumn{7}{|l|}{ Coherence } \\
\hline \multicolumn{7}{|l|}{ F4-T6 } \\
\hline$\delta$ & $0.018 \pm 0.070$ & $-0.088 \pm 0.043$ & 0.002 & 0.029 & 0.057 & 0.208 \\
\hline$\theta$ & $0.037 \pm 0.069$ & $-0.014 \pm 0.061$ & 0.138 & 0.137 & 0.089 & 0.338 \\
\hline$\alpha-1$ & $-0.003 \pm 0.077$ & $-0.020 \pm 0.145$ & 0.761 & 0.505 & 0.820 & 0.110 \\
\hline$\alpha-2$ & $0.003 \pm 0.092$ & $0.005 \pm 0.236$ & 0.805 & 0.784 & 0.361 & 0.124 \\
\hline$\beta-1$ & $0.002 \pm 0.098$ & $0.023 \pm 0.263$ & 0.546 & 0.367 & 0.150 & 0.920 \\
\hline$\beta-2$ & $0.005 \pm 0.133$ & $0.018 \pm 0.222$ & 0.510 & 0.202 & 0.387 & 0.691 \\
\hline \multicolumn{7}{|l|}{$\mathrm{F} 4-\mathrm{O} 2$} \\
\hline$\delta$ & $0.001 \pm 0.058$ & $-0.083 \pm 0.079$ & 0.023 & 0.327 & 0.010 & 0.045 \\
\hline$\theta$ & $0.002 \pm 0.096$ & $-0.003 \pm 0.087$ & 0.053 & 0.081 & 0.005 & 0.521 \\
\hline$\alpha-1$ & $0.026 \pm 0.061$ & $-0.020 \pm 0.167$ & 0.348 & 0.698 & 0.759 & 0.065 \\
\hline$\alpha-2$ & $0.034 \pm 0.080$ & $-0.020 \pm 0.218$ & 0.187 & 0.614 & 0.124 & 0.107 \\
\hline$\beta-1$ & $0.041 \pm 0.092$ & $-0.013 \pm 0.235$ & 0.088 & 0.177 & 0.120 & 0.762 \\
\hline$\beta-2$ & $0.053 \pm 0.115$ & $-0.049 \pm 0.237$ & 0.014 & 0.062 & 0.111 & 0.862 \\
\hline
\end{tabular}

Data (changes in each parameter before and after intervention) were adjusted for gender, fracture site, and age.

were not significantly different between the 2 groups after adjusting for age, fracture sites, and gender.

Pentosidine Levels

Table 2 shows the changes in serum pentosidine levels before and after rehabilitation. Serum pentosidine levels changed significantly in both groups after intervention. As shown in Table 3, however, changes in TDAS did not differ significantly between the 2 groups after adjusting for age, fracture sites, and gender. 
Table 4. Power spectrums before and after intervention

\begin{tabular}{|c|c|c|c|c|c|c|}
\hline & \multicolumn{2}{|c|}{ PT group $(n=16)$} & \multirow[t]{2}{*}{$p$ value } & \multicolumn{2}{|c|}{ PT+OT group $(n=12)$} & \multirow[t]{2}{*}{$p$ value } \\
\hline & before & after & & before & after & \\
\hline \multicolumn{7}{|l|}{$\mathrm{F} 4$} \\
\hline$\delta$ & $0.318 \pm 0.168$ & $0.213 \pm 0.109$ & 0.015 & $0.251 \pm 0.145$ & $0.208 \pm 0.096$ & 0.272 \\
\hline$\theta$ & $0.270 \pm 0.177$ & $0.280 \pm 0.164$ & 0.717 & $0.327 \pm 0.164$ & $0.303 \pm 0.161$ & 0.182 \\
\hline$\alpha-1$ & $0.192 \pm 0.131$ & $0.267 \pm 0.129$ & 0.049 & $0.213 \pm 0.154$ & $0.250 \pm 0.172$ & 0.136 \\
\hline$\alpha-2$ & $0.115 \pm 0.102$ & $0.111 \pm 0.100$ & 0.717 & $0.100 \pm 0.077$ & $0.116 \pm 0.068$ & 0.182 \\
\hline$\beta-1$ & $0.061 \pm 0.046$ & $0.078 \pm 0.042$ & 0.003 & $0.069 \pm 0.038$ & $0.070 \pm 0.030$ & 0.814 \\
\hline$\beta-2$ & $0.044 \pm 0.045$ & $0.051 \pm 0.028$ & 0.017 & $0.039 \pm 0.038$ & $0.053 \pm 0.035$ & 0.158 \\
\hline \multicolumn{7}{|l|}{ F8 } \\
\hline$\delta$ & $0.499 \pm 0.211$ & $0.297 \pm 0.161$ & 0.034 & $0.294 \pm 0.160$ & $0.283 \pm 0.148$ & 0.695 \\
\hline$\theta$ & $0.238 \pm 0.181$ & $0.257 \pm 0.165$ & 0.326 & $0.290 \pm 0.147$ & $0.276 \pm 0.156$ & 0.209 \\
\hline$\alpha-1$ & $0.169 \pm 0.124$ & $0.230 \pm 0.119$ & 0.063 & $0.200 \pm 0.152$ & $0.106 \pm 0.163$ & 0.460 \\
\hline$\alpha-2$ & $0.090 \pm 0.079$ & $0.092 \pm 0.091$ & 0.501 & $0.096 \pm 0.071$ & $0.100 \pm 0.071$ & 0.583 \\
\hline$\beta-1$ & $0.058 \pm 0.043$ & $0.068 \pm 0.029$ & 0.098 & $0.068 \pm 0.031$ & $0.068 \pm 0.024$ & 0.754 \\
\hline$\beta-2$ & $0.046 \pm 0.037$ & $0.056 \pm 0.027$ & 0.056 & $0.051 \pm 0.031$ & $0.056 \pm 0.028$ & 0.583 \\
\hline \multicolumn{7}{|l|}{$\mathrm{T} 4$} \\
\hline$\delta$ & $0.377 \pm 0.145$ & $0.271 \pm 0.112$ & 0.013 & $0.273 \pm 0.137$ & $0.289 \pm 0.142$ & 0.638 \\
\hline$\theta$ & $0.223 \pm 0.138$ & $0.256 \pm 0.163$ & 0.088 & $0.284 \pm 0.146$ & $0.254 \pm 0.129$ & 0.209 \\
\hline$\alpha-1$ & $0.149 \pm 0.105$ & $0.198 \pm 0.094$ & 0.063 & $0.185 \pm 0.130$ & $0.198 \pm 0.138$ & 0.695 \\
\hline$\alpha-2$ & $0.085 \pm 0.051$ & $0.097 \pm 0.069$ & 0.877 & $0.091 \pm 0.068$ & $0.095 \pm 0.060$ & 0.638 \\
\hline$\beta-1$ & $0.080 \pm 0.059$ & $0.092 \pm 0.046$ & 0.215 & $0.072 \pm 0.031$ & $0.075 \pm 0.025$ & 0.754 \\
\hline$\beta-2$ & $0.085 \pm 0.075$ & $0.085 \pm 0.060$ & 0.796 & $0.093 \pm 0.132$ & $0.089 \pm 0.109$ & 0.638 \\
\hline
\end{tabular}

Data are presented as means \pm SD.

\section{EEG Analysis}

Table 4 summarizes the changes in power spectral analysis results after intervention and specifies which sites showed significant differences between the 2 groups. Table 5 provides the results of coherence analysis in each group before and after intervention and shows which fracture sites had significant differences between the 2 groups. As shown in Table 3, an analysis of covariance revealed how age, fracture site, and gender affected the results of both power spectral analysis and coherence analysis.

Power Spectral Analysis

Power values in all frequency bands of all of the 19 electrodes were compared before and after intervention after adjusting for age, fracture sites, and gender. Significant differences in the amount of change of power values of $\delta$ waves on F4, F8, and T4 between the 2 groups. Power spectral analysis of F4, F8, and T4 in each group before and after intervention revealed that the power value of $\delta$ waves decreased significantly at these 3 sites in the PT group after intervention. With the exception of $\theta$ waves and $\alpha-2$ waves, significant differences in power value changes were observed in F4 in the PT group. In the OT group, however, no significant differences in power value changes were noted in these 3 sites after intervention.

Coherence Analysis

Coherence was analyzed in all frequency bands, i.e., F3-F4, 01-02, P3-P4, F3-P3, F4-P4, F4-T6, F3-T5, F3-01, and F4-02, after adjusting for age, fracture site, and gender to compare the 2 treatments. Changes in coherence values for $\delta$ waves in F4-T6 and F4-02 and $\beta-2$ waves of F4-02 after intervention differed significantly between treatments (Table 3). Based on changes in coherence values for F4-T6 and F4-02 after intervention in each group, no signif- 
Table 5. Coherence of before and after intervention

\begin{tabular}{|c|c|c|c|c|c|c|}
\hline \multirow[t]{2}{*}{ Band } & \multicolumn{2}{|c|}{ PT group $(n=16)$} & \multirow[t]{2}{*}{$p$ value } & \multicolumn{2}{|c|}{$\mathrm{PT}+0 \mathrm{~T}$ group $(n=12)$} & \multirow[t]{2}{*}{$p$ value } \\
\hline & before & after & & before & after & \\
\hline \multicolumn{7}{|l|}{ F4-T6 } \\
\hline$\delta$ & $0.260 \pm 0.007$ & $0.251 \pm 0.062$ & 0.776 & $0.326 \pm 0.094$ & $0.269 \pm 0.090$ & 0.003 \\
\hline$\theta$ & $0.230 \pm 0.082$ & $0.251 \pm 0.076$ & 0.587 & $0.271 \pm 0.046$ & $0.279 \pm 0.086$ & 0.969 \\
\hline$\alpha-1$ & $0.208 \pm 0.059$ & $0.200 \pm 0.072$ & 0.587 & $0.234 \pm 0.068$ & $0.222 \pm 0.146$ & 0.456 \\
\hline$\alpha-2$ & $0.216 \pm 0.089$ & $0.219 \pm 0.117$ & 0.816 & $0.254 \pm 0.137$ & $0.259 \pm 0.172$ & 0.784 \\
\hline$\beta-1$ & $0.285 \pm 0.138$ & $0.287 \pm 0.165$ & 0.897 & $0.300 \pm 0.136$ & $0.322 \pm 0.219$ & 1.000 \\
\hline$\beta-2$ & $0.359 \pm 0.146$ & $0.364 \pm 0.174$ & 0.856 & $0.369 \pm 0.114$ & $0.387 \pm 0.212$ & 0.906 \\
\hline \multicolumn{7}{|l|}{$\mathrm{F} 4-02$} \\
\hline$\delta$ & $0.259 \pm 0.059$ & $0.238 \pm 0.050$ & 0.224 & $0.329 \pm 0.132$ & $0.274 \pm 0.076$ & 0.170 \\
\hline$\theta$ & $0.227 \pm 0.083$ & $0.229 \pm 0.057$ & 1.000 & $0.274 \pm 0.075$ & $0.270 \pm 0.089$ & 0.969 \\
\hline$\alpha-1$ & $0.173 \pm 0.059$ & $0.199 \pm 0.084$ & 0.171 & $0.245 \pm 0.081$ & $0.225 \pm 0.151$ & 0.327 \\
\hline$\alpha-2$ & $0.202 \pm 0.085$ & $0.237 \pm 0.124$ & 0.266 & $0.282 \pm 0.136$ & $0.262 \pm 0.177$ & 0.456 \\
\hline$\beta-1$ & $0.269 \pm 0.141$ & $0.311 \pm 0.183$ & 0.093 & $0.344 \pm 0.161$ & $0.331 \pm 0.205$ & 0.844 \\
\hline$\beta-2$ & $0.338 \pm 0.161$ & $0.391 \pm 0.186$ & 0.155 & $0.432 \pm 0.158$ & $0.383 \pm 0.196$ & 0.784 \\
\hline
\end{tabular}

Data are presented as means \pm SD.

icant differences were noted at any frequency band in the PT group after intervention, but there was a significant difference in $\delta$ waves in the PT+OT group after intervention. Change in coherence values in F4-02 increased in all frequency bands in the PT group, whereas it decreased in all frequency bands in the PT+OT group.

\section{Discussion}

TDAS findings showed improved cognitive function in both groups regardless of the rehabilitation method. This was not influenced by age, fracture site, or gender. Regular exercise is effective in improving not only physical fitness but also cognitive functions such as memory, attention, and judgement [27]. Cognitive function improvement may be attributed to the fact that both groups received PT. Decreased serum pentosidine levels suggest that oxidative stress levels decreased after rehabilitation. We believe that moderate exercise increased the secretion of antioxidant enzymes which then prevented oxidative stress [18, 19]. Changes in serum pentosidine levels did not differ significantly between the 2 groups, indicating that both treatments achieved similar antioxidant potentials. As mentioned earlier, pentosidine is the "rust" of bone mass. Decreased levels of "rust" improved bone quality, meaning that the bone strength at hospital discharge had improved compared to before intervention. Also, it was not influenced by age, fracture site, or gender.

Delta waves in F4, F8, and T4, which showed significant intergroup differences in spectral analyses, decreased after intervention in the PT group. In F4, power values increased in almost all frequency bands. These findings suggest that slow-wave activities improved in the PT group. Changes in neurotransmitter levels can alter EEG [11] and, since acetylcholine improves slow-wave activities [28], our results are in line with these findings. Patients in the PT+OT group did not change as much as those in the PT group even though they, too, had received PT.

A previous study showed that electric synchronization in the cerebellar frontal lobe was modulated by GABA [29]. Acetylcholine reinforces both inhibitory and excitatory synapses [30]. Therefore, the significant intergroup difference in F4-02 in the coherence analysis 
suggests that improvements in the PT+OT group were GABA mediated. Since GABA has an inhibitory effect, this may account for why power values did not increase as much in the $\mathrm{PT}+\mathrm{OT}$ group as in the PT group. F4-02 forms a long loop and preceding research shows that lower frequencies correspond to longer circulation times and longer loop distances [31]. Thus, changes in $\delta$ wave synchronization in F4-02 observed in our study are in line with these previous findings. Synchronization of $\delta$ waves not only implies that neurons connect over long distances, but also that this intrahemispheric coherence is an important manifestation of neurocognitive networking [31]. The significant intergroup difference in $\beta-2$ waves in F4-02 we observed supports this theory. Neurons continuously exposed to external stimulation adapt to the environment by decreasing in number due to synaptic structural changes [32]. Since cognitive function improved in the PT+OT group despite interelectrode coherence values that were significantly lower than the PT group after intervention, our results also support this theory. Coherence values of $\delta$ waves in F4-T6 also differed significantly between the 2 groups, suggesting long-term inhibition in the prefrontal region-thalamus-cerebellum area of the right hemisphere in the $\mathrm{PT}+\mathrm{OT}$ group may have engendered synaptic plasticity. Although the coherence values at F4-02 and F4-T6 in the PT group did not change significantly, they increased at almost all frequency bands. Since cognitive function improved after intervention in the PT group, long-term potentiation may have produced synaptic plasticity. Older people are generally known to exhibit compensatory cerebral activity in an attempt to preserve motor functions that have deteriorated with aging [33]. Preceding research estimates that the cortical source of single task activities is in the left hemisphere, whereas that of dual task activities is in the right hemisphere [34]. Serrien et al. [35] discussed shifts in the dominance of the 2 hemispheres during different tasks and noted that the left hemisphere was considered to be dominant for motor behavior in right-handed individuals. However, they also stated that lateralization of motor function is indeed flexible and driven by several factors. The right hemisphere specializes in spatial functions, including spatial attention, and it has been proposed that the relative involvement of each hemisphere in a task depends on that task's specific characteristics [34]. Applying this theory to our study, we now understand that the cortical source shifted from the left hemisphere to the right hemisphere. Though EEG power and coherence were said to differ by age and gender in a previous study [36], the effect of rehabilitation was hardly influenced by age, fracture site, or gender.

The cerebellum was long thought to execute motor function, but it is currently considered to play a role in cognitive function, especially in the cognitive aspects of behavior [37]. Longterm potentiation of cerebral synaptic transmission efficiency enables us to remember knowledge that can be explained in words, whereas long-term inhibition promotes cerebellar learning that can be acquired through experience [38].

This study shows that rehabilitation with both PT and PT+OT has the same positive effect on cognitive function and antioxidant potential. However, EEG findings suggest that each form of rehabilitation therapy has a different effect on brain neurons.

To prevent refractures in a subsequent fall, body balance and posture must be maintained. This requires a continuous inflow of information starting with viewing of the surroundings, acknowledgement of the space, and body movement [33], which can be realized by the neuronal connection between the frontal lobe, the thalamus, and the cerebellum. $\mathrm{PT}+\mathrm{OT}$ rehabilitation may be effective in regaining this ability within the limited timespan of a hospital stay. More cases must be studied to further verify the effects of rehabilitation on brain function in patients with fractures. 


\section{Acknowledgement}

We thank all of the patients who participated in our study as well as their families. We also thank all of the staff at Yukoukai Kaikeonsen Hospital (Yonago, Japan) who cooperated in this study.

\section{Statement of Ethics}

All of the subjects were thoroughly informed of the objectives and methods of this study and submitted a written informed consent form before enrollment. This study was approved by the Institutional Review Board of the Faculty of Medicine of Tottori University.

\section{Disclosure Statement}

The authors have no conflict of interests to declare.

\section{Funding Sources}

This research did not receive any specific grant from funding agencies in the public, commercial, or not-for-profit sector.

\section{Author Contributions}

N.M., K.M., S.M, K.M., and K.U. contributed to the conception and design of this study, data acquisition, and analysis/interpretation of the data. N.M., K.M., S.M, K.M., and K.U. contributed to the conduction of this study and made substantial intellectual contributions to the drafting of this or figures. All of the authors approved the final version of this paper.

\section{References}

1 Bridenbaugh SA, Kressig RW. Motor cognitive dual tasking: early detection of gait impairment, fall risk and cognitive decline. Z Gerontol Geriatr. 2015 Jan;48(1):15-21.

2 Tinetti ME, Speechley M, Ginter SF. Risk factors for falls among elderly persons living in the community. N Engl J Med. 1988 Dec;319(26):1701-7.

3 Barnett A, Smith B, Lord SR, Williams M, Baumand A. Community-based group exercise improves balance and reduces falls in at-risk older people: a randomised controlled trial. Age Ageing. 2003 Jul;32(4):407-14.

4 Yoshimura N, Muraki S, Oka H, Mabuchi A, En-Yo Y, Yoshida M, et al. Prevalence of knee osteoarthritis, lumbar spondylosis, and osteoporosis in Japanese men and women: the research on osteoarthritis/osteoporosis against disability study. J Bone Miner Metab. 2009;27(5):620-8.

5 Yoshimura N, Muraki S, Nakamura K, Tanaka S. Epidemiology of the locomotive syndrome: the research on osteoarthritis/osteoporosis against disability study 2005-2015. Mod Rheumatol. 2017 Jan;27(1):1-7.

6 Kendler DL, Bauer DC, Davison KS, Dian L, Hanley DA, Harris ST, et al. Vertebral Fractures: Clinical Importance and Management. Am J Med. 2016 Feb;129(2):221.e1-10.

7 Hagino H. Fragility fracture prevention: review from a Japanese perspective. Yonago Acta Med. 2012 Jun; 55(2):21-8.

8 Baker NL, Cook MN, Arrighi HM, Bullock R. Hip fracture risk and subsequent mortality among Alzheimer's disease patients in the United Kingdom, 1988-2007. Age Ageing. 2011 Jan;40(1):49-54.

9 Itou Y, Nochi R, Kuribayashi H, Saito Y, Hisatsune T. Cholinergic activation of hippocampal neural stem cells in aged dentate gyrus. Hippocampus. 2011 Apr;21(4):446-59. 
10 Chen N, Sugihara H, Sur M. An acetylcholine-activated microcircuit drives temporal dynamics of cortical activity. Nat Neurosci. 2015 Jun;18(6):892-902.

11 Başar E, Düzgün A. How is the brain working?: research on brain oscillations and connectivities in a new "takeoff" state. Int J Psychophysiol. 2016 May;103:3-11.

12 Wong FK, Bercsenyi K, Sreenivasan V, Portalés A, Fernández-Otero M, Marín O. Pyramidal cell regulation of interneuron survival sculpts cortical networks. Nature. 2018 May;557(7707):668-73.

13 Babiloni C, Lizio R, Marzano N, Capotosto P, Soricelli A, Triggiani AI, et al. Brain neural synchronization and functional coupling in Alzheimer's disease as revealed by resting state EEG rhythms. Int J Psychophysiol. 2016 May;103:88-102.

14 Jelic V, Johansson SE, Almkvist O, Shigeta M, Julin P, Nordberg A, et al. Quantitative electroencephalography in mild cognitive impairment: longitudinal changes and possible prediction of Alzheimer's disease. Neurobiol Aging. 2000 Jul-Aug;21(4):533-40.

15 Fleck JI, Kuti J, Brown J, Mahon JR, Gayda-Chelder C. Frontal-posterior coherence and cognitive function in older adults. Int J Psychophysiol. 2016 Dec;110:217-30.

16 Bonda DJ, Wang X, Lee HG, Smith MA, Perry G, Zhu X. Neuronal failure in Alzheimer's disease: a view through the oxidative stress looking-glass. Neurosci Bull. 2014 Apr;30(2):243-52.

17 Ott C, Jacobs K, Haucke E, Navarrete Santos A, Grune T, Simm A. Role of advanced glycation end products in cellular signaling. Redox Biol. 2014 Jan;2:411-29.

18 Hitomi Y, Watanabe S, Kizaki T, Sakurai T, Takemasa T, Haga S, et al. Acute exercise increases expression of extracellular superoxide dismutase in skeletal muscle and the aorta. Redox Rep. 2008;13(5):213-6.

19 Hatao H, Oh-ishi S, Itoh M, Leeuwenburgh C, Ohno H, Ookawara T, et al. Effects of acute exercise on lung antioxidant enzymes in young and old rats. Mech Ageing Dev. 2006 Apr;127(4):384-90.

20 Halliwell B, Gutteridge JMC. Free radicals in biology and medicine. 4th ed. Oxford: Oxford University Press; 2015

21 Karim L, Vashishth D. Heterogeneous glycation of cancellous bone and its association with bone quality and fragility. PLoS One. 2012;7(4):e35047.

22 Karim L, Tang SY, Sroga GE, Vashishth D. Differences in non-enzymatic glycation and collagen cross-links between human cortical and cancellous bone. Osteoporos Int. 2013 Sep;24(9):2441-7.

23 Inoue M, Jimbo D, Taniguchi M, Urakami K. Touch Panel-type Dementia Assessment Scale: a new computerbased rating scale for Alzheimer's disease. Psychogeriatrics. 2011 Mar;11(1):28-33.

24 Rosen WG, Mohs RC, Davis KL. A new rating scale for Alzheimer's disease. Am J Psychiatry. 1984 Nov; 141(11): 1356-64.

25 Kogan EA, Korczyn AD, Virchovsky RG, Klimovizky SSh, Treves TA, Neufeld MY. EEG changes during long-term treatment with donepezil in Alzheimer's disease patients. J Neural Transm (Vienna). 2001;108(10):1167-73.

26 Snaedal J, Johannesson GH, Gudmundsson TE, Blin NP, Emilsdottir AL, Einarsson B, et al. Diagnostic accuracy of statistical pattern recognition of electroencephalogram registration in evaluation of cognitive impairment and dementia. Dement Geriatr Cogn Disord. 2012;34(1):51-60.

27 Erickson KI, Voss MW, Prakash RS, Basak C, Szabo A, Chaddock L, et al. Exercise training increases size of hippocampus and improves memory. Proc Natl Acad Sci USA. 2011 Feb;108(7):3017-22.

28 Kondo S, Kawaguchi Y. Slow synchronized bursts of inhibitory postsynaptic currents (0.1-0.3 Hz) by cholinergic stimulation in the rat frontal cortex in vitro. Neuroscience. 2001;107(4):551-60.

29 Du X, Rowland LM, Summerfelt A, Choa FS, Wittenberg GF, Wisner K, et al. Cerebellar-Stimulation Evoked Prefrontal Electrical Synchrony Is Modulated by GABA. Cerebellum. 2018 May;17(5):550-63.

30 Mitsushima D, Sano A, Takahashi T. A cholinergic trigger drives learning-induced plasticity at hippocampal synapses. Nat Commun. 2013;4(1):2760.

31 Bressler SL, Tognoli E. Operational principles of neurocognitive networks. Int J Psychophysiol. 2006 May; 60(2):139-48.

32 Sugie A, Hakeda-Suzuki S, Suzuki E, Silies M, Shimozono M, Möhl C, et al. Molecular Remodeling of the Presynaptic Active Zone of Drosophila Photoreceptors via Activity-Dependent Feedback. Neuron. 2015 May;86(3): 711-25.

33 Santos Monteiro T, Beets IA, Boisgontier MP, Gooijers J, Pauwels L, Chalavi S, et al. Relative cortico-subcortical shift in brain activity but preserved training-induced neural modulation in older adults during bimanual motor learning. Neurobiol Aging. 2017 Oct;58:54-67.

34 Bogost MD, Burgos PI, Little CE, Woollacott MH, Dalton BH. Electrocortical sources related to whole-body surface translations during a single- and dual-task paradigm. Front Hum Neurosci. 2016 Oct; 10:524.

35 Serrien DJ, Ivry RB, Swinnen SP. Dynamics of hemispheric specialization and integration in the context of motor control. Nat Rev Neurosci. 2006 Feb;7(2):160-6.

36 Kober SE, Reichert JL, Neuper C, Wood G. Interactive effects of age and gender on EEG power and coherence during a short-term memory task in middle-aged adults. Neurobiol Aging. 2016 Apr;40:127-37.

37 Lu X, Miyachi S, Takada M. Anatomical evidence for the involvement of medial cerebellar output from the interpositus nuclei in cognitive functions. Proc Natl Acad Sci USA. 2012 Nov; 109(46):18980-4.

38 Ito M, Yamaguchi K, Nagao S, Yamazaki T. Long-term depression as a model of cerebellar plasticity. Prog Brain Res. 2014;210:1-30. 